


\title{
Progettare la «Stimmung». Dialogo tra Quintus Miller e Armando Ruinelli
}

\author{
Design the «Stimmung». Dialogue between Quintus Miller \\ and Armando Ruinelli
}

Edited by Anna Innocenti

\begin{abstract}
The architects Quintus Miller and Armando Ruinelli operate mainly in Switzerland and in particular in the Grisons area where they have carried out several projects thus facing the different issues affecting the requalification of landscape and of existing architecture in the valley and mountain context.

The dialogue between the two architects highlights their design approach in relation to the historical, cultural and environmental peculiarities of this heritage. What emerges strongly is the need for the contemporary project to reinterpret the existing in order to identify and restore in each project the «Stimmung» intended as an absolute synthesis of all those elements that characterize a given place or a given architecture in time and space.

From the architectural redevelopment of small buildings to the insertion of new volumes within historical fabrics, from the restoration of monuments to the expansion of historic structures, the narrated projects show a well-read approach towards the intervention on heritage allowing a critical reinterpretation of history, of memory and of the long lasting Alpine settlement processes.
\end{abstract}

\section{Quintus Miller}

Born in 1961, architect ETH SIA BSA graduated, together with Paola Maranta in 1990 in Basel he founded the studio Miller \& Maranta with which they received numerous awards including the RIBA International Fellowship in London in 2012.

He has been visiting professor at the EPF in Lausanne and at the Polytechnic University of Zurich and he has been full professor at the Mendrisio Academy of Architecture since 2009.

\section{Armando Ruinelli}

Born in Sondrio in 1954, he is BSA architect, in 2000 he founded Ruinelli Associati SIA Architects with Fernando Giovanoli.

\section{Anna Innocenti}

Born in Sondrio in 1984, she has been architect at Ruinelli Associati since 2011.
Keywords

Heritage, Alps, contemporary

architecture, infill, renovation. 
AR: Quando affronti un progetto in zone alpine, hai un approccio diverso rispetto a quando operi in città?

QM: Per me non c'è differenza. Il lavoro dell'architetto è creare uno spazio per l'essere umano, anticipare una situazione di vita e dargli una forma architettonica e urbanistica che sembra appropriata. Adotto un processo quasi uguale, tra un progetto e un altro. Parto da una ricerca sul sito e sul contesto, sul tema del progetto e sul cliente. Lì si manifesta una prima differenza, perché il cittadino e il montanaro sono tra loro diversi. Tuttavia ritengo che anche i paesi alpini, come Soglio o gli altri nuclei nei Grigioni, abbiano aspetti molto urbani. Al pari delle grandi città come Basilea o Parigi, anche qui c'è uno spazio pubblico, c'è una struttura gerarchica che è espressione e rappresentazione della società che l'ha prodotta. E questo è dappertutto paragonabile.

AR: In effetti, mi aspettavo questa risposta, cui mi collego per la prossima domanda.

È un pensiero condiviso che tutte le città devono rinnovarsi, altrimenti muoiono.

Mentre nell'opinione di molte persone c'è l'idea che i nuclei nelle Alpi debbano essere conservati per una sorta di "bisogno romantico".

Come ti confronti con la tesi del rinnovo dei piccoli nuclei?

QM: Questo è un tema molto delicato. La società è cambiata, i paesi nelle montagne non sono più i paesi che servono ai bisogni dei contadini, sono diventati paesi che si offrono ai turisti. Il desiderio del cittadino che viene in questi paesi è "romantico", vuole ritrovare ricordi e avere un contatto con la natura, il materiale, con gli odori della campagna che non si trovano più nelle città. Tuttavia, gli abitanti di questi nuclei alpini hanno la volontà e il diritto di vivere nel contemporaneo.

In apertura

Restauro e

ampliamento

Villa Garbald,

Miller\&Maranta,

2002-2005,

Bregaglia, Grigioni,

Svizzera (foto Ruedi

Walti).
Posso citare l'esempio dell'albergo Waldhaus a Sils, dove lavoriamo da quasi trent'anni.

Io sono cresciuto in un albergo nelle Alpi svizzere e so quel che l'ospite si aspetta normalmente. Vuole riconoscere sempre il "suo" albergo. L'atmosfera non deve cambiare troppo, ma è opportuno migliorare sempre perché se l'albergo non si adatta al tempo a un certo punto non funziona più.

Nei nostri interventi ci sono nuovi elementi, ma ci sono sempre anche aspetti che s'integrano nell'atmosfera complessiva.

L'intervento sul bar è stato enorme, tutto è stato demolito, soletta e pavimento compreso. Abbiamo spostato il bancone in modo da creare un asse nell'edificio e generare un percorso per l'ospite, che parte dal bar, si reca al ristorante attraversando la hall, e al bar ritorna dopo la cena. È possibile riconoscere tutto ciò che è originale, come il rivestimento delle pareti, le integrazioni, come la ricostruzione della boiserie intorno alle finestre, e gli elementi completamente nuovi, come il bancone del bar e le lampade. Abbiamo anche cambiato il colore, questo bar era verde, ora è di un rosso scuro. Di giorno può funzionare come ristorante con la grande finestra verso il bosco, la sera è completamente un altro ambiente e il rosso gli conferisce un'atmosfera più scura, più forte, da bar nel senso classico inglese. Tuttavia la Stimmung è coerente con le altre parti dell'albergo. Per noi questa continuità era molto importante.

L'abbiamo ricercata anche nel fumoir. Abbiamo utilizzato lo stesso materiale per i rivestimenti, il noce europeo, così che si senta una familiarità tra i due ambienti, mentre lo spazio ha una geometria nuova. Il fumoir ha la forma di un uovo, senza angoli, con solo tre aperture: la porta d'entrata, la finestra sulla roccia e il camino. Il camino ha una referenza con la tipologia storica, è alimentato a legna, è rivestito in ceramica verde e si trova a pavimento. La finestra s'affaccia sulla roccia, come se fosse una fotografia in bianconero senza cornice. Tutto concorre a rendere lo spazio accogliente, uno spazio in cui riunirsi.

Ritengo dunque che l'architettura, a tutte le scale, debba cercare di stabilire rapporti con un determinato contesto e sapersi integrare, attraverso la novità del contemporaneo. 


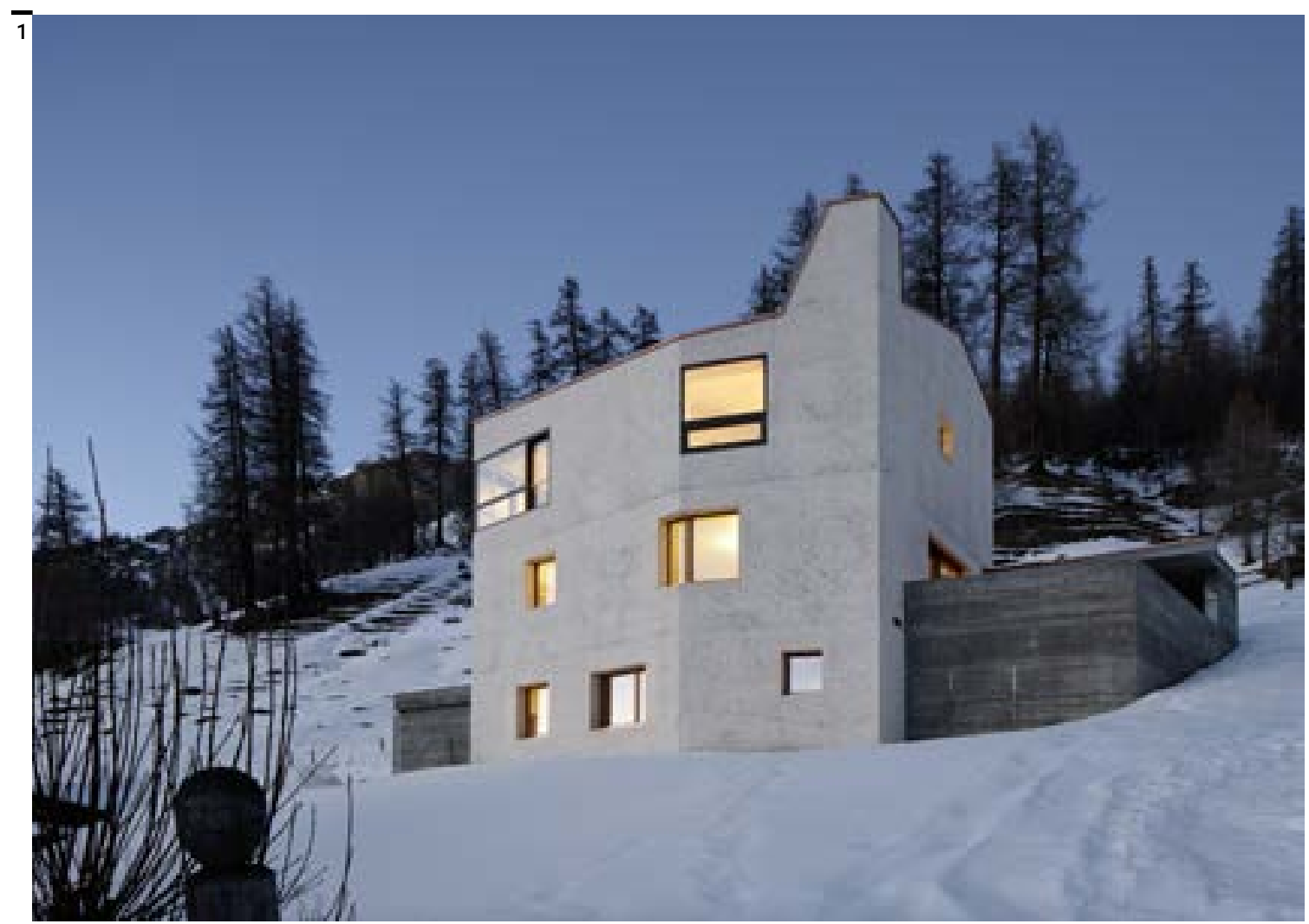

Fig. 1

Casa unifamiliare

in Val Monastero,

Ruinelli Associati

Architetti, 2014 (foto

Ralph Feiner)

Fig. 2

\section{$\overline{2}$}

\section{$\overline{2}$}

Garbald

- Scientist

in residence,

Castasegna, Ruinelli

Associati Architetti,

2019 (foto Marcello

Mariana).

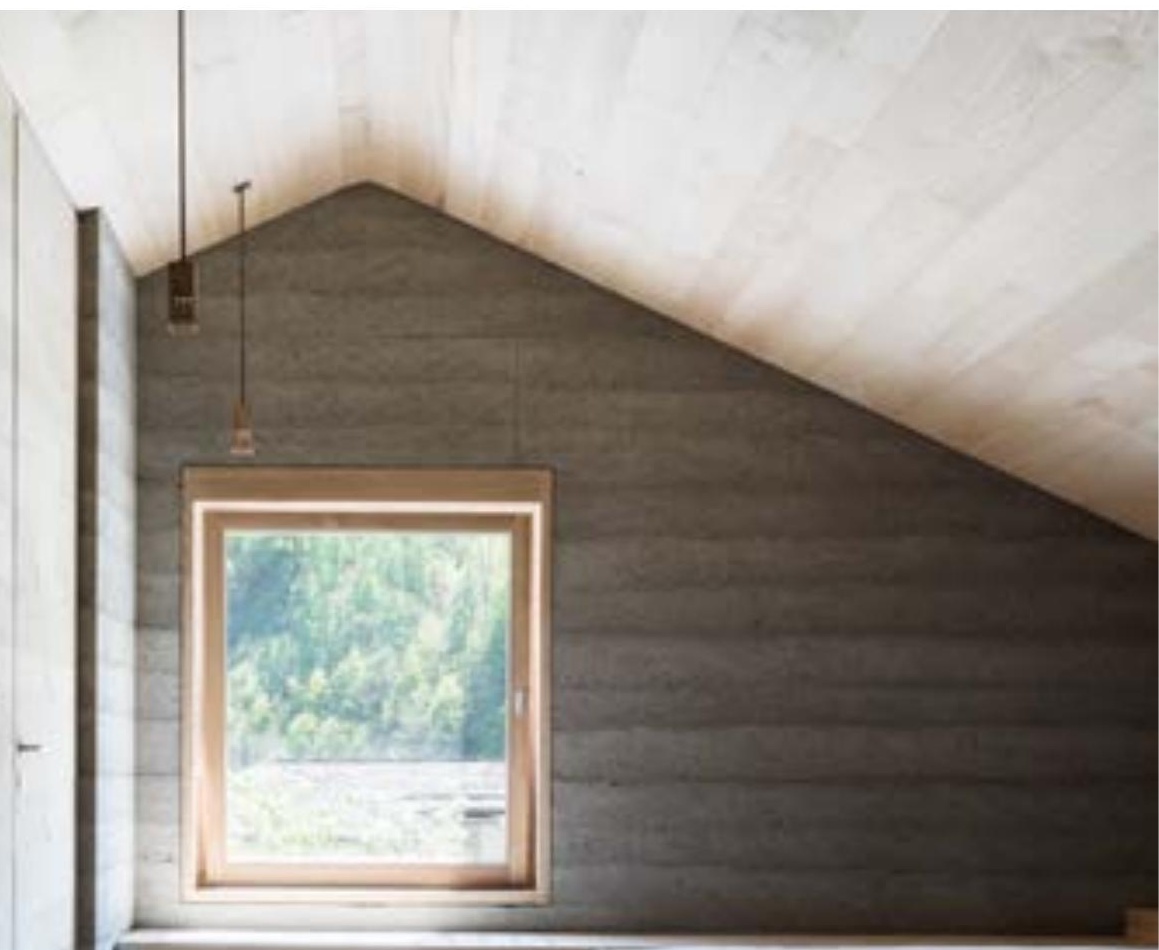


AR: Nei piccoli paesi, come Soglio, che è un esempio emblematico di raggruppamento, il modo di costruire così chiuso e compatto ha portato a una recente dispersione insediativa attuata specialmente dagli indigeni che hanno costruito la casa fuori, intorno al nucleo.

Tale dispersione cambia l'immagine del territorio e degli insediamenti, con un continuo consumo di suolo, da una parte, e l'abbandono dei nuclei, dall'altra.

Quando la Bregaglia ha ricevuto il premio Wakker ho iniziato a ragionare su una possibile dispersione interna ai nuclei. Cosa ne pensi della tesi di sfoltire i nuclei per invertire la tendenza a costruire fuori da essi? Quale potrebbe essere il destino delle stalle, dentro i nuclei, che ora non sono più utilizzate? Potrebbero esserci altri approcci, oltre alla loro trasformazione in case di vacanze - operazione tra l'altro non più praticabile nei Grigioni - come ad esempio togliere alcuni edifici per creare una piazza o un giardino pensile, conferendo nuova qualità e luce ai nuclei? Oppure ritieni che sia meglio non intervenire?

QM: Io penso che questi paesi debbano trovare un modo di continuare a vivere e non diventare musei di se stessi. La mia posizione è progressista, il cambiamento è necessario, ma perché si possa proiettare questi paesi nel futuro, è necessario un progetto, un tema, un masterplan, che non riguarda solo l'urbanistica e l'architettura, ma anche l'economia. Si devono creare le opportunità e l'attrattività per far sì che un numero sufficiente di persone vi risieda, paghi le tasse e che il comune possa sopravvivere.

Nel passato di questi paesi c'era una sorta di common sense su come si doveva vivere, lavorare e costruire. Questo senso collettivo è stato tradotto in tipologie, materiali e metodi costruttivi, che hanno formato un insieme, un carattere particolare, ancora oggi leggibile nella sua forza.

Perciò quando si vogliono introdurre dei cambiamenti, si dovrebbe avere una übergeordnete Idee, un approccio complessivo, che permetta la comprensione dell'insieme.

Le stalle in disuso potrebbero essere demolite e sostituite, ma sempre con l'idea di ricostruire un insieme. Per esempio si potrebbero sostituire con case di legno, con una determinata tipologia e costruzione, che ridefiniscono una nuova unità. $\mathrm{Al}-$ cuni palazzi e case rimangono, come testimoni della storia, e il contemporaneo deve trovare il modo di integrarsi, con garbo e adeguatezza, con grande rispetto per l'esistente.

L'integrazione non deve avvenire soltanto su un piano formale, ma anche di contenuto, di significati.
AR: $\grave{E}$ analogo al discorso sul Waldhaus a Sils-Maria, cioè cambiare senza che ci si accorga, in altre parole, non ri-scrivere la storia, ma continuare a scriverla.

QM: Sono d'accordo. La storia non deve essere percepita "contro" il contemporaneo, ma la contemporaneità è essa stessa parte della storia. Per me è importante che il patrimonio storico e l'intervento contemporaneo creino un insieme in cui si possano comprendere le parti. Questo è ciò che ha guidato il nostro progetto dell'Ospizio del San Gottardo, in cui il tema era ricostruire un edificio dando una nuova forma e facendo l'interno quasi tutto nuovo, in legno, mantenendo una coerenza dell'insieme.

AR: Nel tuo processo progettuale, quando subentra la riflessione sui materiali?

QM: Immediatamente. Quando affronto un progetto, costruzione e materiale sono fin dal primo momento soggetti importanti. Come architetti anticipiamo certe Stimmungen e le costruiamo con argomenti architettonici, le proporzioni, i materiali, la luce.

Senza pensare alla materializzazione, non riesco a immaginare lo spazio e l'emozione che può procurare ai suoi abitanti.

L'architettura deve sempre avere un aspetto di emozionalità, perché è fatta per esseri umani e anche per non-architetti. Per me è importante che anche un non-architetto riesca a leggere un mio edificio, a usarlo e a comprenderne il vero significato, anche se a un livello subcosciente. Ciò mi riesce solo se suscito delle emozioni.

AI: Nell'ambito della Fondazione Garbald a Castasegna ci sono tre vostri interventi: il restauro di un monumento, l'inserimento di un'architettura nuova e la trasformazione di un edificio della cultura materiale. Vorrei chiedervi un commento dei progetti, con particolare riferimento alla possibilità per l'architettura contemporanea di generare - o rigenerare - il tessuto urbano in cui è inserita.

AR: Il nostro intervento è su una cascina nel prato dietro al complesso della villa Garbald. Il regolamento edilizio ha imposto la ricostruzione con posizione, volume e altezza identici all'edificio preesistente.

In verità, non solo non abbiamo potuto, ma nemmeno abbiamo voluto agire diversamente. Le dimensioni molto ridotte dell'edificio e il contesto in cui sorge, richiedevano un approccio pacato, con pochi segni contemporanei, come ad esempio le gronde con i canali in calcestruzzo. Il nostro progetto affronta temi della storia, pensata con i mezzi costruttivi di oggi, e ha a che fare con i materiali. 
È un intervento che reinterpreta l'esistente con rigore e che non ha la necessità di esprimersi con un altro volume, secondo il detto: "Un tetto piano non qualifica un'architettura come contemporanea".

QM: Concordo con Armando sul fatto che rimanere entro delle regole, permette al suo progetto di funzionare benissimo.
Anche un piccolo nucleo, come Castasegna, esprime l'essenza dell'urbano, cioè il fatto che gli uomini hanno capito che vivere insieme rende la società più efficace. La città occidentale funziona come una massa anonima su cui la politica imprime una struttura, attraverso assi, strade, monumenti. La qualità è data dall'insieme e anche dall'anonimità. Quando l'architettura contemporanea s'impone come monumento di se stessa e interviene sull'esi-
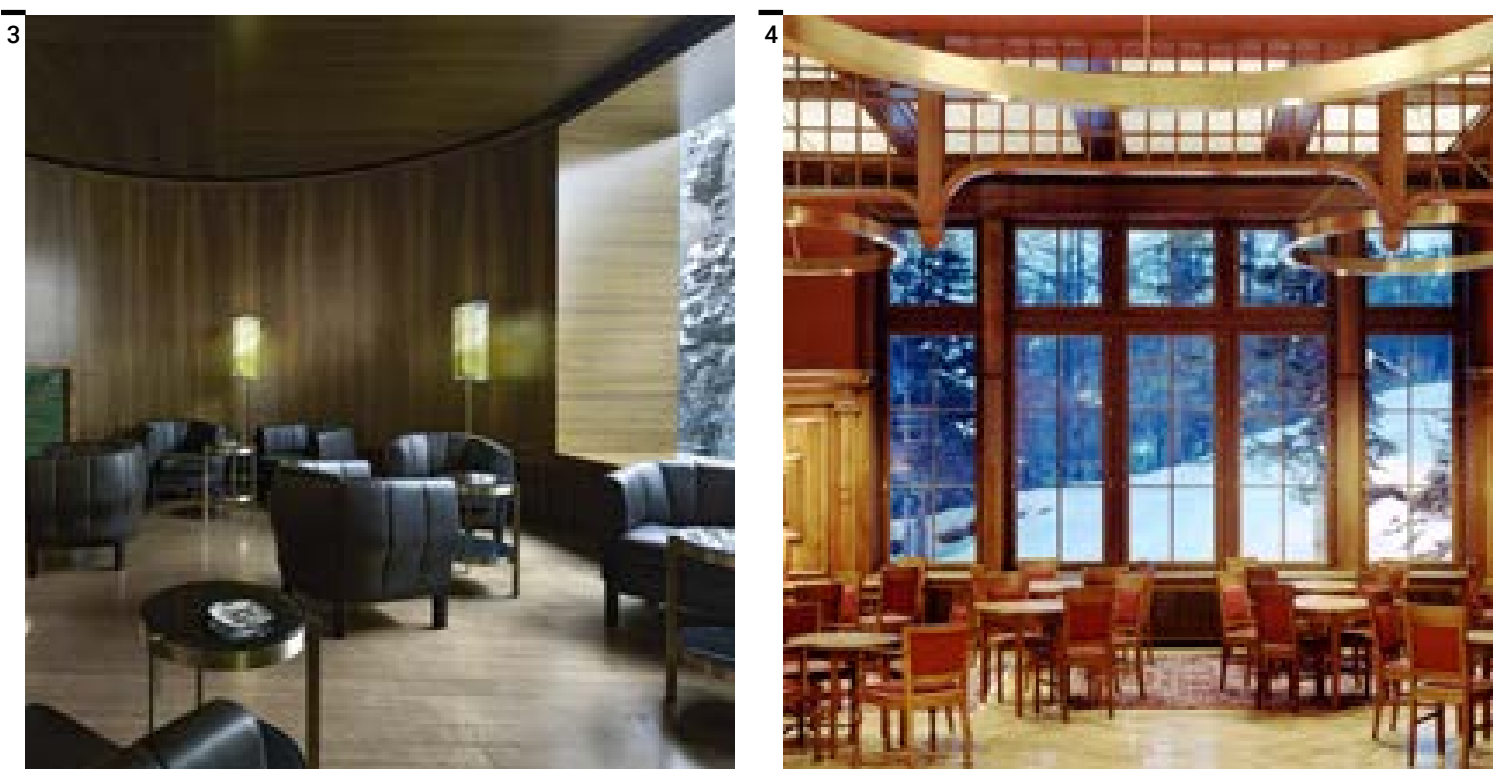

Figg. 3-4

Conversione e

ampliamento

Hotel Waldhaus,

Miller\&Maranta,

2016, Sils im

Engadin, Grigioni,

Svizzera (foto Ruedi

Walti).

Fig. 5

Edificio residenziale

Schwarzpark,

Miller\&Maranta,

2004, Basilea,

Svizzera (foto Ruedi

Walti).
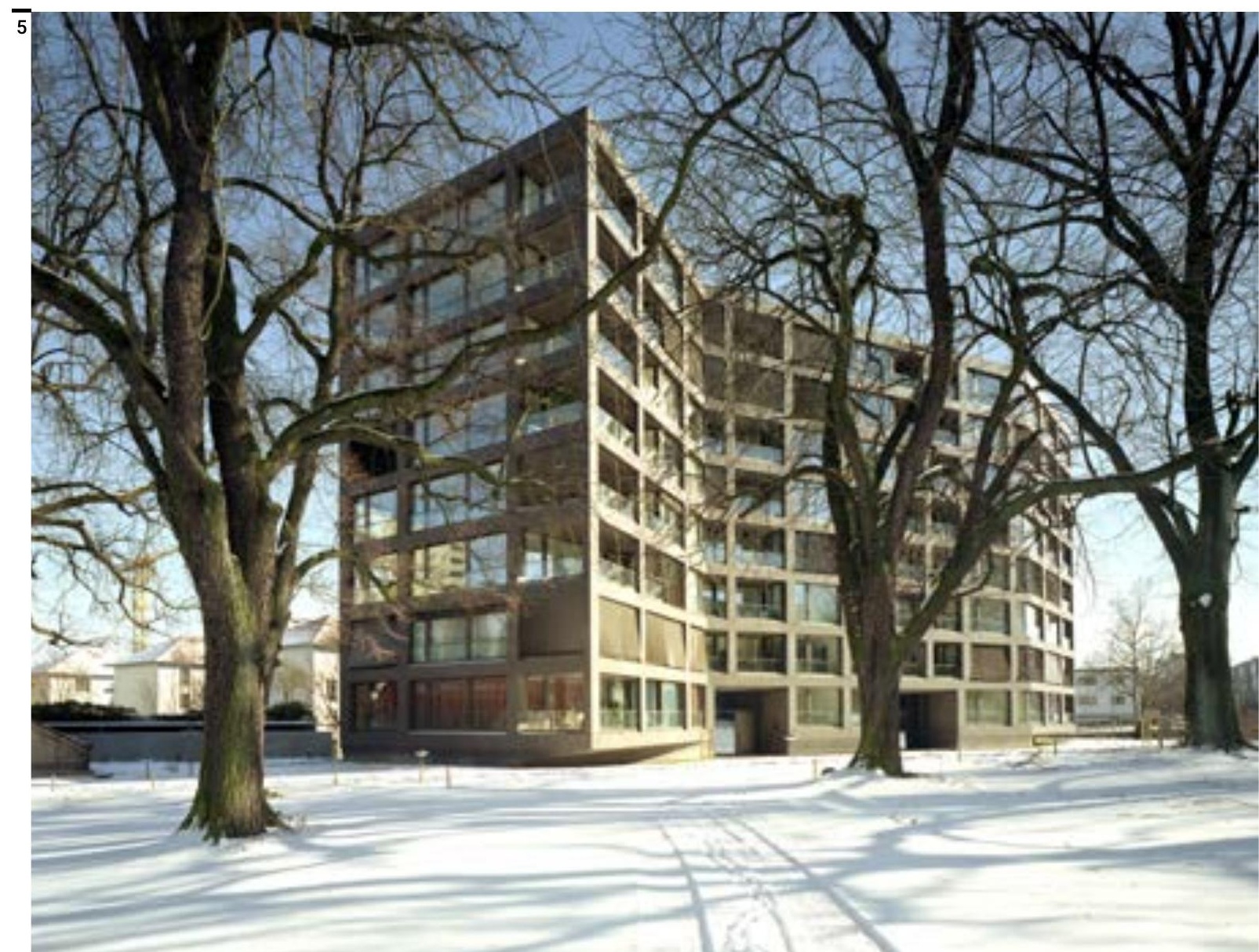
stente con oggetti autonomi, distrugge il vero senso dell'insieme. La città delle architetture individuali diventa una cacofonia.

Per me la prima cosa da valutare è il contesto e l'insieme, nella sua essenza, e la reinterpretazione con i mezzi di oggi. È necessario che l'uso dei materiali e le tecniche costruttive siano attuali e onesti, solo con quest'approccio la Stimmung riesce a essere costante.

Quando abbiamo progettato il Roccolo, per noi era importante costruire una casa che non fosse una villa. Il nostro edificio occupa una posizione in "secondo piano", vi si accede attraverso il giardino della villa di Semper, come fosse un edificio rurale, e si sviluppa con un'altra tipologia ed espressività.

Con questi argomenti abbiamo cercato di equilibrare la nuova costruzione con la villa e di integrarla nel tessuto urbano. Quando si guarda dall'altra parte della valle, socchiudendo gli occhi, non si coglie che c'è un edificio nuovo perché la proporzione, le aperture, il materiale, s'integrano nel contesto.

Quando ci si avvicina, si capisce che è un edificio del 2000, ma il suo carattere gli permette di interagire con l'intorno, ha una certa "familiarità" con il paese. Il materiale grezzo della facciata rinvia ai muri che orlano le strade, alle stalle e alle costruzioni rurali. L'altezza è simile alle case tutt'intorno. Attraverso la precisione del dettaglio e la lettura dei materiali abbiamo cercato di porci in relazione con il capolavoro di Semper, provando a tradurre il suo pensiero nel nuovo edificio. Per esempio le finestre sono tagliate nella facciata molto ruvida e sono lisce nell'imbotte.

\section{AR: ì interessante che questa torre non abbia una facciata principale, come invece possiede la villa. È come se crescesse fuori dal muro del giardino. \\ QM: Proprio questa era la nostra volontà, la torre declina il medesimo linguaggio del muro. La pian- ta ha una punta che crea una diagonale nel giardi- no, in direzione dell'uscita dalla valle, così da non stabilire un rapporto frontale con la villa. La vil- la rimane autonoma, le permettiamo di essere let- ta nel suo significato originale.}

\section{AI: Che cosa vi stupisce dei luoghi diversi da quelli in cui operate abitualmente?}

AR: Due anni fa abbiamo costruito una casa nel Meckleburg-Vorpommern. Ciò che mi lasciava perplesso, a bocca aperta, di quei luoghi era la $S e^{-}$ elandschaft, le erbe lunghe e secche che qui noi non conosciamo, il loro movimento nel vento, la luce diffusa e la sensazione di non riuscire a dare un limite ad un territorio che appare sconfinato.
In un'altra situazione, in Val Monastero, ho progettato un edificio fuori dal villaggio. Ho avuto difficoltà perché, per la prima volta, non avevo elementi costruiti, edifici o muri, a cui appoggiarmi. Nel progetto sono allora subentrati altri aspetti, il terreno, la forma dei prati, gli abeti che in Bregaglia non sono vicini alle case.

Queste sono le prime sensazioni che mi colpiscono e mi danno un'immagine del luogo, non di cosa progetterò, ma di una Stimmung.

QM: Io ho una grande curiosità per le cose, quando vado in un luogo nuovo, ho tutti i sensi all'erta, provo a capire e a combinare la percezione con le mie memorie per cogliere il significato e l'essenza di un luogo.

Attualmente stiamo lavorando a dei progetti a Brema e ad Amburgo. Di queste città mi stupiscono le dimensioni, le proporzioni, il classicismo, l'eco della storia delle grandi capitali del nord e degli uomini che sono andati per mare.

Ho iniziato ad interessarmi al Giappone perché volevo guardare da fuori alla nostra cultura e, attraverso la differenza, coglierne i significati profondi. Lo scorso ottobre ho insegnato e vissuto a Kyoto. In Giappone è tutto diverso e io ero sempre fuori dalle regole. Il confronto con un'altra cultura $\mathrm{mi}$ ha fatto capire ancor meglio quanto sia importante la memoria per il nostro mestiere.

La percezione non può avvenire senza memoria, tutto è legato a essa. Anche la memoria dell'ambiente costruito è fondamentale. Potrei affermare che l'architettura stessa è memoria, perché il costruito è memoria della cultura umana. Come architetto anticipo una situazione attraverso la mia memoria e posso contare sulla memoria collettiva così che questa situazione abbia un significato per la società.

\section{AI: Entrambi avete parlato di Stimmung. Potete darne una definizione, dato che il termine non ha una traduzione univoca in italiano?}

QM: Stimmung è un modo di vedere lo spazio, legato alla memoria. Si potrebbe tradurre in altre lingue con il termine "atmosfera". Ma "atmosfera" non è abbastanza precisa, ha sempre qualcosa d'indeterminato. Stimmung è un termine germanico, che si riferisce allo spazio ma anche alla musica, all'accordare uno strumento. Il verbo stimmen è anche legato all'avverbio es stimmt, "è giusto". Accordare significa allora "fare che lo strumento sia giusto".

AR: Stimmung è un concetto molto vasto. Rappresenta l'atmosfera, l'emozione, il formalmente giusto. È la sintesi di tutti questi elementi che, se sono esatti l'uno con l'altro, crea Stimmung. In italia- 


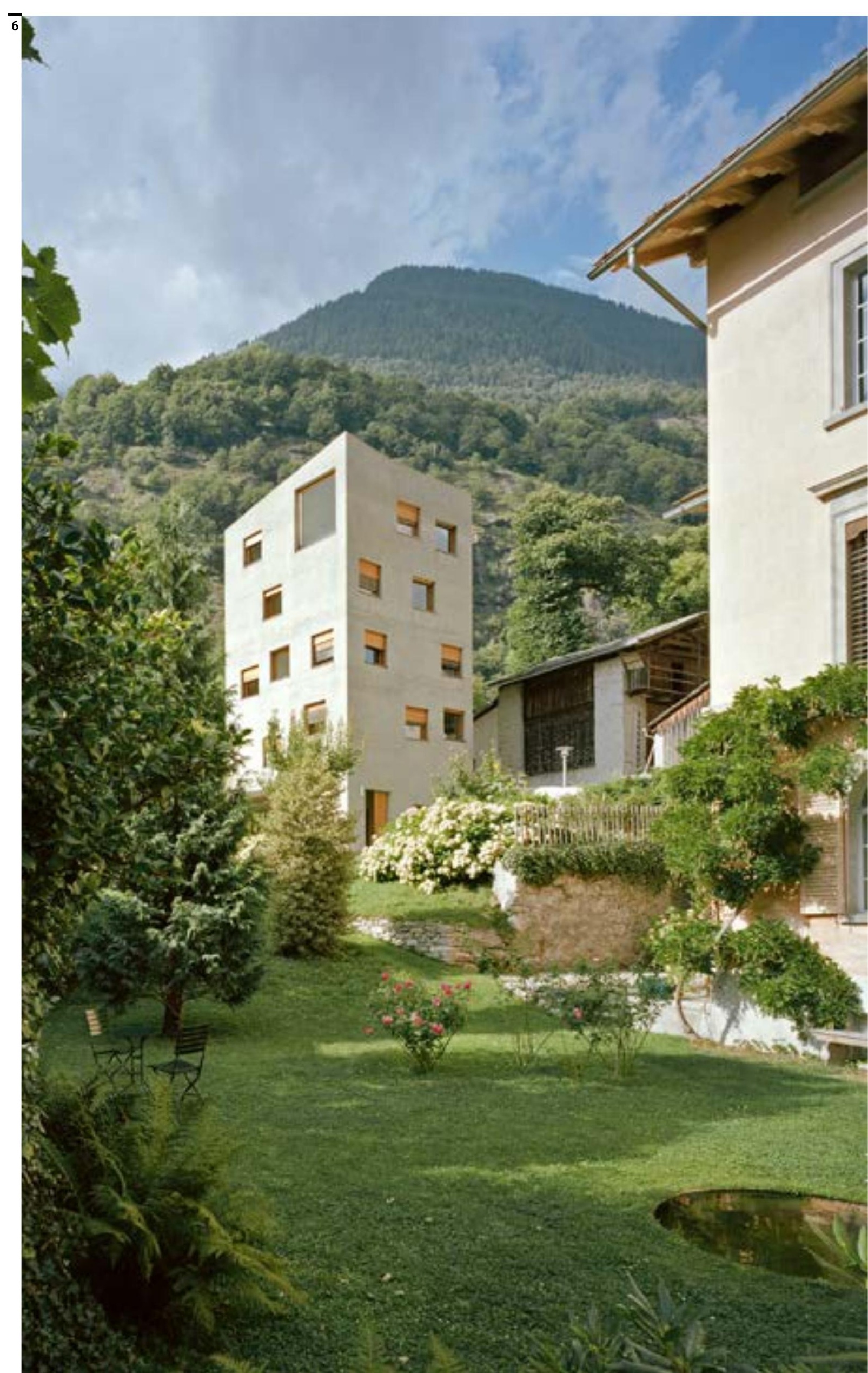

Restauro

ampliament

Villa Garbald,

Miller\&Maranta,

2002-2005

Bregaglia, Grigioni,

Svizzera (foto Ruedi

Walti). 
no dovremmo spiegarla in singoli pezzi, come un puzzle. Mentre l'emozione e l'atmosfera sono più vaghe, la Stimmung è precisa. È anche "fattibile".

QM: In architettura, Stimmung si può definire attraverso argomenti, legati alla memoria personale e alla coscienza collettiva. La Stimmung si può costruire con argomenti architettonici, attraverso proporzioni, materiali, luce, colori.

Nella mia formazione all'ETH di Zurigo è stato fondamentale l'insegnamento dell'Architettura Analoga, che utilizzava l'immagine come uno strumento di rappresentazione emozionale dello spazio. Ho imparato che in architettura c'è sempre anche un aspetto emozionale, che lo spazio ha in sé qualcosa di più della sola logica intellettuale, che l'importanza della memoria non è solo la coscienza, ma anche il sotto-cosciente.

Per me l'architettura deve essere multistrato e avere un ventaglio di significati, così che essa possa rimanere rilevante per un tempo futuro. M'interessa poter introdurre dei cambiamenti ai significati condivisi, magari a livello sotto-cosciente, in modo che non sia tutto ovvio a prima vista. Tuttavia, sono consapevole che questi estraniamenti sono delicati da fare e richiedono il massimo di responsabilità.

Dieci anni fa abbiamo costruito un condominio in un parco, lo Schwarzpark a Basilea. L'idea è stata di progettare l'edificio come se fosse un ulteriore albero situato nel parco protetto, con grandi querce. Uno scenario molto bello in cui abbiamo cercato di costruire con rispetto, integrando l'edificio nel verde.

Abbiamo pensato a una struttura di calcestruzzo per la facciata, con una combinazione di prefabbricazione, per ragioni di economia, e di getto sul

Fig. 7

Torre Heuwaage,

Miller\&Maranta,

2017, Basilea,

Svizzera (render

Filippo Bolognese).

Fig. 8

Casa FE, Ruinelli

Associati SA.

Meclemburgo-

Pomerania Anteriore,

Germania (foto

Marcello Mariana)

Fig. 9

Riqualificazione stalla, Ruinelli

Associati SA., 2009,

Soglio, Grigioni,

Svizzera (foto

Marcello Mariana) o in Germania. Per capire il perché, è necessario fare alcune premesse.

Nella Svizzera del dopoguerra l'ingegneria aveva una grande importanza. Era diffuso il pensiero del "tutto è possibile". Era la cultura del "fattibile", dell'homo faber. Questo concetto fu descritto da Max Frisch nel suo romanzo intitolato Homo faber. In quest'opera anticipa la dissoluzione, il fallimento della società del "tutto fattibile" all'inizio

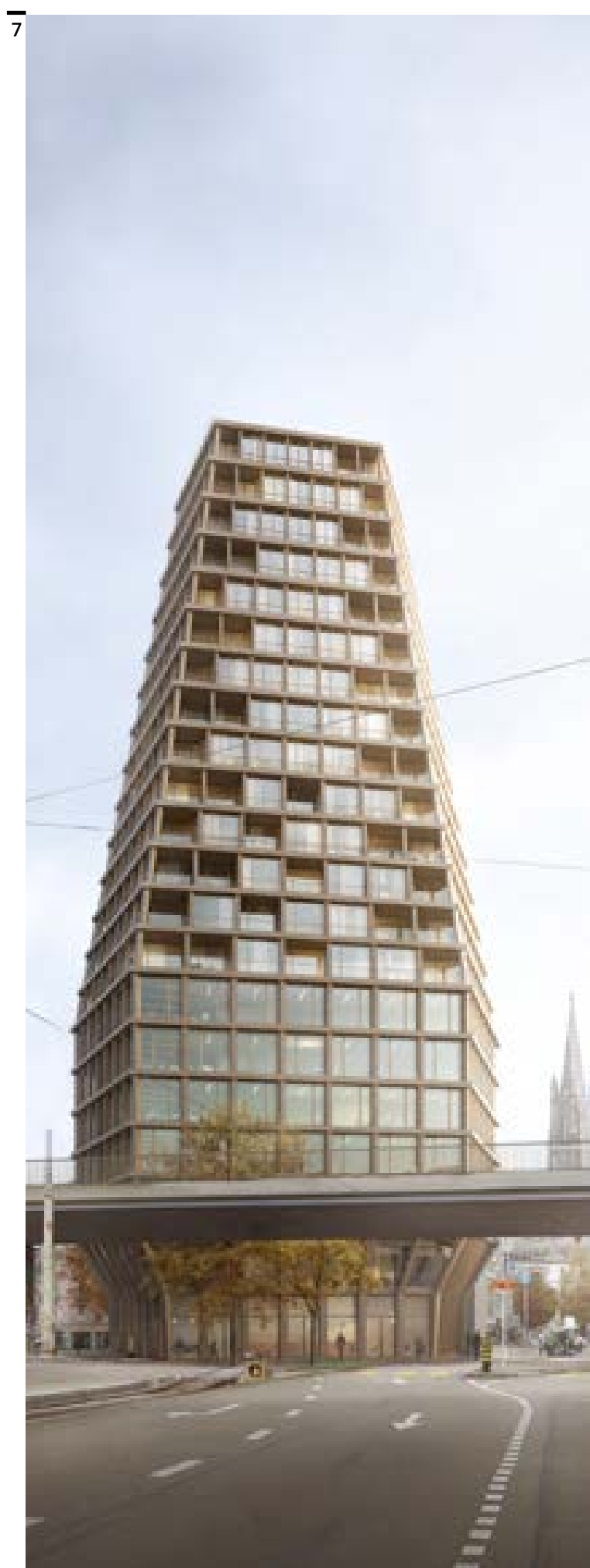
montate verticalmente, sono leggermente inclinate, per coprire completamente l'apertura, composta da finestra e parapetto vetrato. Questa inclinazione non si nota subito, ma guardando bene, c'è qualcosa d'inaspettato, che irrita per la prospettiva cambiata e invita a guardare due volte.

Questo è un aspetto che m'interessa, progettare un'architettura che non sia ovvia, ma che forzi la gente a guardare con attenzione, per essere compresa.

AI: Aldo Rossi viene da voi più volte citato tra le vostre referenze. Quanto è fondativa la lettura tipologica urbana per il vostro modo di progettare?

QM: Aldo Rossi ha insegnato a Zurigo all'ETH, da lui hanno studiato molti architetti oggi conosciuti. In Svizzera Rossi ha avuto un influsso molto importante, diverso da quello riscontrato in Italia 
degli anni Settanta, dove tutto cambia. È l'inizio del postmodernismo, in cui si capisce che la rottura con la storia magari è stata giusta nel $1920 \mathrm{e}$ dopo, ma nel 1970 non è più appropriata. È necessario fermarsi e trovare un altro rapporto con la terra e con la storia.

In Svizzera in questi anni ci sono la creazione della legge per la tutela dei monumenti e la stesura delle Raumplanungsgesetz, che regolano le zone edificabili.
In questo contesto, Aldo Rossi ha insegnato all'ETH. Nei suoi corsi ha fatto disegnare agli studenti una pianta della città di Zurigo simile alla pianta Nolli di Roma, con il piano terra degli edifici storici, dove si vede il rapporto degli edifici allo spazio. Si capisce che attraverso i secoli si è formato un tessuto che crea una comunità attuale $\mathrm{e}$ nel quale la storia è integrata. Questo pensiero di Aldo Rossi ha trovato una grande risonanza, non a

\section{$\overline{8}$}
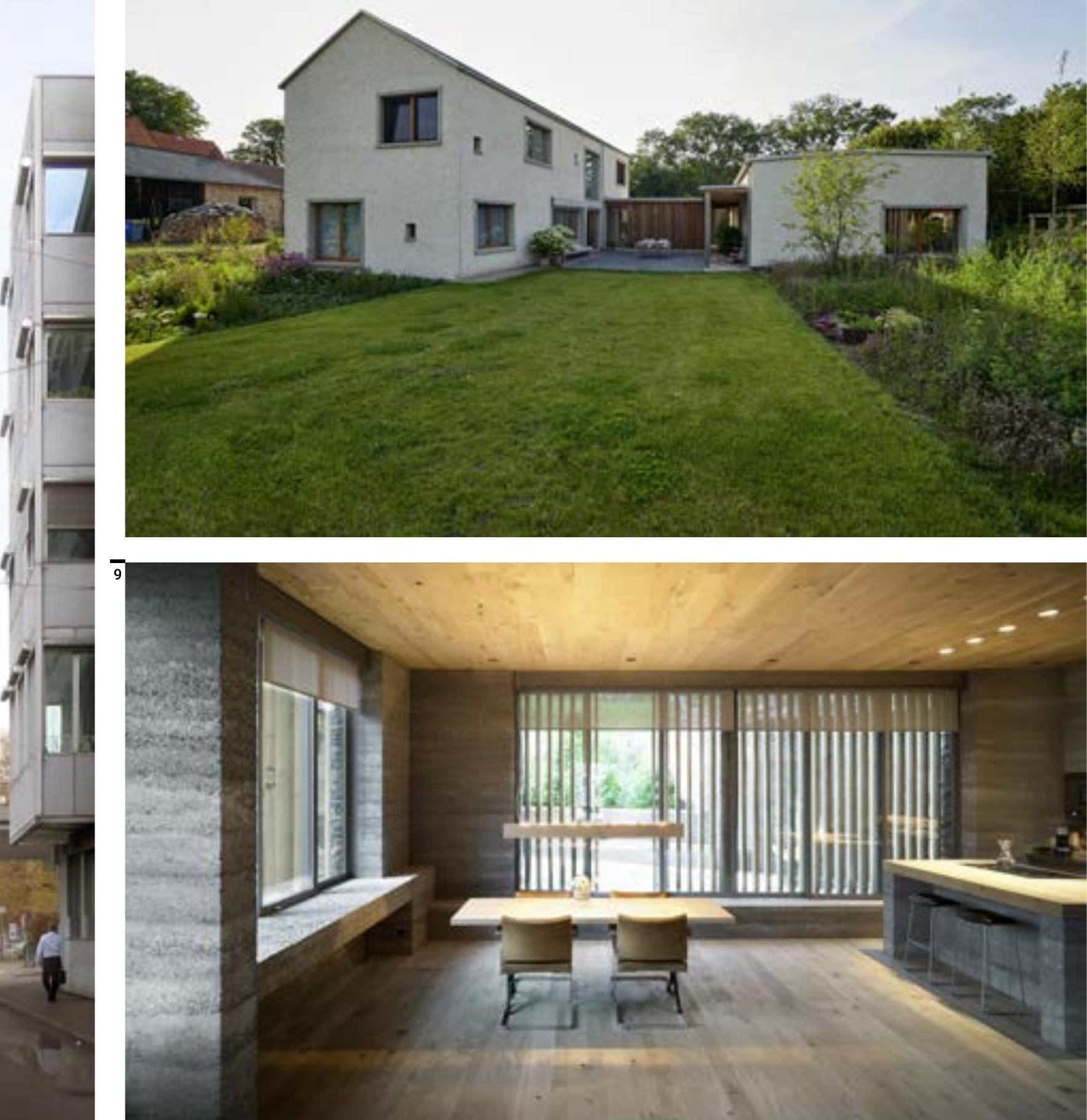
livello dell'immagine delle sue architetture, ma del significato del suo insegnamento.

L'invenzione dell'architetto non è più "io creo un oggetto indipendente", ma bisogna leggere la città, capire la storia e integrarla nel pensiero progettuale.

AR: Ritengo che questo suo insegnamento sia stato più importante rispetto alla sua architettura, che oggi ci appare con tutti i limiti del post-modernismo.

Io ho imparato la sua lezione sulla piccola scala, attraverso Michael Alder. Anche un villaggio richiede una lettura tipologica per poterne comprendere la morfologia e i caratteri.

QM: Io ho conosciuto il pensiero di Rossi attraverso Fabio Reinhart e il suo assistente Miroslav Sik. Quando ho studiato da loro, ho dovuto lavorare con referenze, che non erano gli architetti razionalisti, ma per esempio il lavoro di Asplund, le architetture ottocentesche, il classicismo. In questo contesto ho cominciato a pensare l'architettura in funzione di Stimmung. Noi studenti ci esercitavamo a comporre delle grandi rappresentazioni, con pastelli a olio, delle vere pitture, per creare situazioni architettoniche dotate di una certa atmosfera, con luce, nebbia, sole, luna, sperimentando su un livello emozionale.

Per la generazione degli architetti svizzeri cinquantenni di adesso, che sono stati in grande numero studenti di Reinhart e Šik, questo è l'influsso di Aldo Rossi: non l'aspetto post-moderno del collage di architetture storiche, ma il tema di integrare la storia nel pensiero e nella strategia progettuale, per la costruzione di un nuovo luogo.

\section{AR: Vorresti aggiungere un commento a un tuo progetto in corso?}

QM: Attualmente stiamo lavorando alla torre Heuwaage a Basilea. Il progetto ha vinto un concorso indetto nel 2017 per la costruzione di una torre al bordo della città storica.

Per ragioni urbanistiche abbiamo liberato la torre dal tessuto urbano esistente, così da poterne cogliere l'autonomia. Lo studio sulla luce ci ha condotto verso una forma, la cui comprensione non è immediata. La nuova torre si relaziona con la ricostruzione ottocentesca della città, emblematica nella chiesa Elisabethen, neogotica, presente nelle vicinanze. Questa strategia è paragonabile al rapporto tra la

Figg. 10-12 Rinnovo e ampliamento del Rifugio

San Gottardo, Miller\&Maranta, 2010, Passo San Gottardo, Svizzera (foto Ruedi Walti).
Torra Velasca di BBPR e il Duomo di Milano, che si nota in tante immagini d'epoca in cui, sullo sfondo della torre si vedono le guglie del Duomo. Abbiamo pensato la facciata della nostra torre in calcestruzzo, senza fughe, in modo che avesse un aspetto massiccio. L'interpretazione della torre è quindi il continuare a costruire la città in modo contemporaneo ma sempre con significati contestuali.

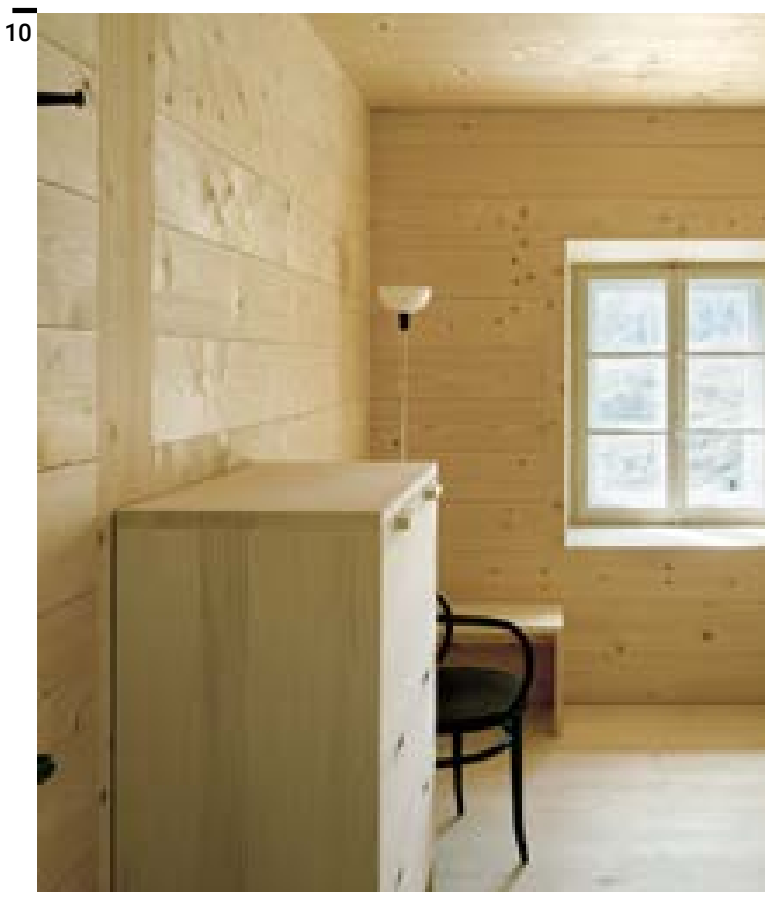

$\overline{12}$ 

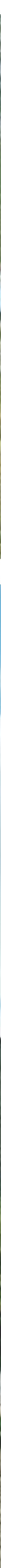